\title{
Automation of Complex Operational Scenarios - Providing 24/7 Inter-Satellite Links with EDRS
}

\author{
Thorsten Beck, Michael Schmidhuber and Jan-Christoph Scharringhausen \\ Deutsches Zentrum für Luft- und Raumfahrt e. V., German Aerospace Center \\ Münchener Straße 20, 82234 Weßling, Germany
}

\begin{abstract}
The European Data Relay System (EDRS) offers a high-speed communication service between ground stations and LEO satellites, using a relay satellite constellation in geostationary orbit. This paper presents the overall system design of the EDRS Payload Control Center, located at the German Space Operation Center (GSOC), with a focus on automation components developed in-house and their integration into the GSOC multi-mission environment.
\end{abstract}

\section{Introduction}

The European Data Relay System (EDRS) consists of two geostationary satellites, EDRS-A and EDRS-C, and a ground station network dedicated to the provision of a high-speed communication service to operators of low-Earth orbit (LEO) spacecraft and unmanned aerial vehicles (UAVs). EDRS-A was launched in February 2016 and is currently in its commissioning phase. It is positioned in geostationary orbit, $9^{\circ}$ East, and is equipped with a state-of-the-art Laser Communication Terminal (LCT) and Ka-band relay payload. EDRS-C is foreseen to be launched in 2017 and will be positioned $31^{\circ}$ East.

The EDRS project arose from the need to increase contact times and communication data rates between LEO satellites and their respective ground station network. Typical contact intervals of a LEO satellite are of the order of 10 minutes, which is all the time available for a continuous downlink of user data and upload of mission time-lines, before contact is lost over one particular site. The task of managing ground stations around the world and scheduling contact times is a complex coordination effort and a considerable cost factor for a satellite operator's budget.

This is where the European Data Relay System comes in. EDRS-A, from its geostationary position, is able to increase the visibility of LEO satellites over the European hemisphere up to intervals of around 45 minutes. The LCTs installed on EDRS-A and -C allow optical inter-satellite links with data rates up to 1800 MBit per second, an increase by a factor of 3.5 when compared to conventional X-band downlink rates. EDRS-A additionally hosts a Ka-band payload, allowing RF inter-satellite links with up to 300 MBit per second, usable by LEO satellites which are not equipped with an LCT counter terminal. ${ }^{1}$

As part of the ESA ARTES 7 program, EDRS is realized as a public private partnership with Airbus DS as prime contractor and DLR (German Aerospace Center) responsible for major parts of the ground network, including the implementation and operation of the Devolved Payload Control Center (DPCC) and the Satellite Control Center (SCC) in case of EDRS-C.

Due to the commercial nature of the program, highly ambitious performance requirements have been defined and need to be met by the DPCC: the service level agreement foresees continuous payload utilization, up to 200 links per day and communication channel, over the expected lifetime of 15 years, with a targeted 


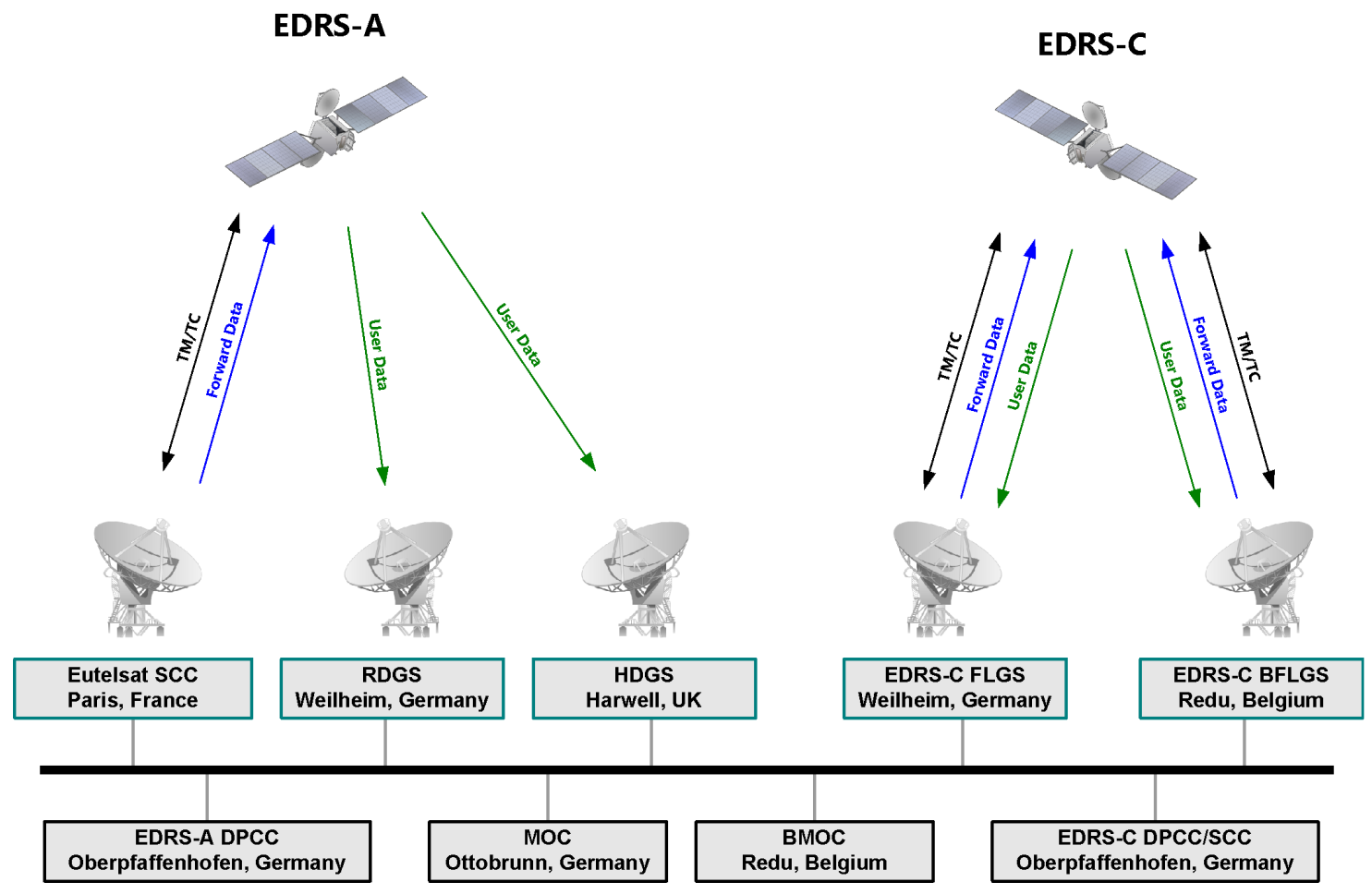

Figure 1: EDRS ground network

service availability of at least $99.6 \%$ over 60 days and an order reaction time well below one hour; a task that can hardly be handled in a manual or semi-automated operations concept.

The EDRS payload is therefore controlled using a fully-automated operations engine which complements GSOCs core monitoring and control system (MCS), an enhanced derivative of ESAs SCOS-2000 v3.1. The automation engine is designed to supervise the complete cycle of telecommand uplink and execution, as well as reaction monitoring of telemetry. Updates to the onboard mission timeline are scheduled and up-linked autonomously, triggered either by spacecraft events or high-level external link requests. Telemetry analysis and key performance indicators are provided in quasi real-time to the Mission Operations Center (MOC).

With EDRS-C being hosted on a very different platform than EDRS-A, the DPCC ground segment was designed to be independent of the particular spacecraft platform. This is realized via a layered system architecture centered around the core MCS, with layer 1 consisting of the commanding front-end and automation engine while layer 2 comprises the Link Management System (LMS) and interface to MOC. This layered architecture allows for a seamless phase-in of EDRS-C, with no software changes required for layer 2 and only minor upgrades to components of layer 1 . Differences in the platform will be masked through the versatility of the core MCS.

\section{Ground Segment Design}

As it is characteristic for European projects, EDRS involves a number of parties from different national organizations and industry partners. The Mission Operations Center (MOC) is operated by Airbus Defense and Space and located in Ottobrunn, Germany. The MOC is responsible for the coordination of user requests, service availability analysis and high-level link planning, which serves as the basis for the actual scheduling and commanding of inter-satellite link requests.

For EDRS-A, the satellite control center (SCC) is operated by Eutelsat with the SCC located in Paris. Eutelsat is responsible for operating the satellite during launch and early orbit phase (LEOP) and for all 
platform related activities during routine operations. Commanding of the payload must be routed through Eutelsat SCC and the SCC reports the telecommand uplink status back to DPCC.

For EDRS-C, the ground segment setup is more conventional in that the satellite control center is colocated with the payload control center, with DLR being responsible for both satellite platform and payload operations.

\section{A. Devolved Payload Control Center}

The devolved payload control center (DPCC) for EDRS-A and EDRS-C is located at the DLR German Space Operation Center (GSOC). It is responsible for operating the optical and RF payload during routine operations and, if necessary, during contingency operations. To better understand the considerations that were incorporated into the ground segment design, we shall briefly review the satellite platforms and commanding concepts of EDRS-A and EDRS-C in more detail:

The space segment of EDRS-A is realized as a hosted payload onboard the Eutelsat-EB9B satellite. The EB9B platform is based on the Airbus DS Eurostar 3000 platform, a CCSDS compatible spacecraft bus providing frame-based telemetry. It is in use for over 50 active geostationary communication satellites. The LCT payload on the other hand provides packet based telemetry in accordance to the more contemporary ECSS Packet Utilization Standard (PUS), ${ }^{2}$ which is embedded in the host telemetry stream.

For EDRS-A, the DPCC receives and processes the full telemetry stream, including platform telemetry. Commanding of the Ka- and optical payload has to be forwarded via file interface to the SCC, where the commands are then routed through their own MCS for uplink to the spacecraft.

EDRS-C on the other hand is a satellite dedicated almost exclusively to the EDRS mission, with some additional space reserved for hosted payloads. It is based on the very recent small-GEO platform, a fully PUS-compliant spacecraft platform for geostationary communication satellites developed by OHB as part of the ESA Artes 11 program.

The EDRS-C telemetry is received via ground-stations in Weilheim, Germany and Redu, Belgium. Commanding is done via the same route and since the DPCC is co-located with the EDRS-C SCC, there is no need for a file interface: the DPCC interfaces directly with the EDRS-C command and control system.

The main drivers in the design of the DPCC ground segment can be summarized as follows:

(a) A single system design for the payload control center, applicable to both EDRS-A and EDRS-C, despite the differences in satellite platform and commanding concepts.

(b) Fully autonomous routine operations to manage the high level of payload utilization, with no need for continuous operator supervision.

(c) Seamless integration in the existing multi-mission environment and reuse of already available software components, as far as possible.

(d) Ease of system updates and maintenance without service interruption over the complete operational lifetime of 15 years.

\section{B. Layered Architecture}

From a data handling point of view, the differences in both EDRS platforms are considerable and require the use of a versatile monitoring and control system. The GSOC monitoring and control system, which is based on ESA SCOS-2000 3.1 and goes by the name of GECCOS, ${ }^{3}$ has been adapted over the last decade to support a wide range of missions and diverse spacecraft platforms. 
Additional adaptations are necessary, not only on the level of operational products such as flight procedures and TM-TC databases, but also on the level of DPCC software components. Since every software adaptation binds resources and poses high demands on component verification and validation, one of the main goals of the preliminary design was the separation of components that are completely reusable for both missions, differing only in their configuration, and systems that cannot avoid changes to their implementation.

Eventually, a layered architecture centered around the core MCS system was defined where layer 1 components need to undergo changes in implementation and layer 2 components are adapted to EDRS-C via configuration change only. A simplified schematic of the layered DPCC architecture is presented in Figure 2.

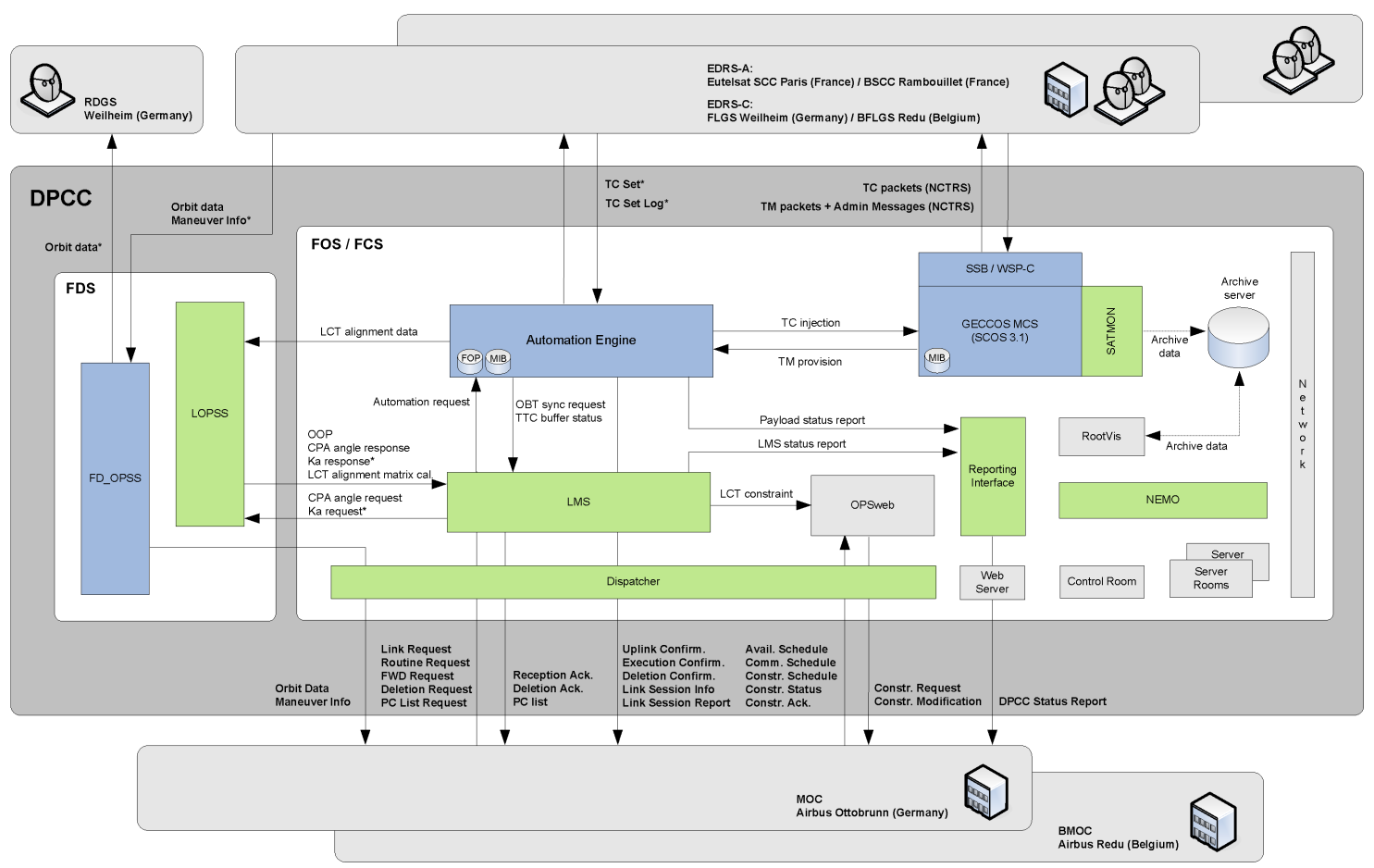

Figure 2: DPCC functional architecture and interfaces. Blue components belong to layer 1, green components to layer 2. Interfaces marked with an asterisk are required only for EDRS-A.

\section{The GSOC Multi-Mission Concept}

The DPCC is part of the DLR GSOC multi-mission control center. The multi-mission aspect is realized through broad and comprehensive training of the operations team and a commonality of systems across different projects. This includes the use of common user interfaces and applications. Many core GSOC software components have evolved over time and were adapted to mission-specific requirements while maintaining a consistent user interface.

The second aspect of the multi-mission approach is the deployment of virtualization concepts for computing platforms. This process has been implemented at GSOC over the last years, with EDRS being one of the first missions that derives full advantage from the concept. The achieved goals include:

(a) the ability to freely move positions or mission environments among control rooms

(b) the ability to upgrade and replace hardware independent of the installation

Control room positions are therefore equipped with thin clients, applicable for every mission environment. The mission specific virtual machines are hosted on ESXi Servers, with their desktop environment being 
forwarded using the NX protocol for Unix based and RDP for Windows based environments. Servers, firewalls, switches and routers are physically located in two different locations, thereby avoiding single point of failures.

The virtualization approach is supplemented by a central software package management, automated deployment mechanisms and configuration management, using open source tools like Puppet. Both concepts together highly streamline the process of deployment, testing and configuration control. ${ }^{5}$

\section{Automation of EDRS-A Routine Operations}

Although a large part of the link request management, in particular all user interactions, is handled by the Mission Operations Center, the DPCC has full responsibility over the satellite payload and has been entrusted with the implementation of all operational products, i. e. ground and flight procedures, and a major part of the software solutions enabling a continuous service availability.

The DPCC routine activities fall into two categories: nominal activities related to the provision of the link service, based on high-level requests from the Mission Operations Center. Secondly, the management of payload configuration and maintenance activities, which must be scheduled in coordination with any external requests. Both types of activities are executed in a fully autonomous way by the Link Management System and automation engine, designed and developed by DLR GSOC for the EDRS mission.

\section{A. Payload Configuration}

The interface between MOC and DPCC foresees five types of fully automated and one semi-automated payload requests:

1. Payload Link Configuration: a high-level link request, containing information about the designated payload (EDRS-A/EDRS-C, Ka or optical), link type (forward or return), data rate and link timings. It also contains information about the targeted LEO orbit which needs to be processed by the flight dynamics system (FDS) before it can be uplinked to the spacecraft. The DPCC is responsible for automatically preparing the payload in the correct configuration such that the requested link can be executed successfully.

2. Payload Routine Configuration: a straight-forward request for execution of a predefined Flight Operations Procedure (FOP). The set of routine procedures that can be requested in this way has been agreed upon between MOC and DPCC beforehand. The request must contain all necessary parameter values required for instantiation of the flight procedure.

3. Forward Tasking Data: this request type prepares the system for a link in forward direction, uploading data from ground to the LEO satellite. The request contains binary data which is uplinked and buffered inside the onboard data processing unit (DPU).

4. Payload Configuration Deletion: a deletion request that removes a scheduled payload link or routine configuration from the mission time-line, either on ground or onboard in case it was already commanded to the spacecraft.

5. Forward Tasking Data Deletion: a deletion request with the goal of clearing the onboard DPU from forward data, previously uplinked via Forward Tasking Data requests.

6. Payload Basic Configuration: similar to routine requests, but executed manually by DPCC payload experts and operators. This type of request typically requires some level of coordination between MOC and DPCC, the actual parameters and execution times are agreed upon during this process.

These requests are sent via file interface from MOC to DPCC where they are processed by the Link Management System and the automation engine. 


\section{B. Link Management}

The Link Management System is responsible for automated scheduling and management of configuration and deletion requests received from the Mission Operations Center (MOC). It is based on the existing GSOC planning and scheduling software suite, complemented by EDRS-specific interface components. ${ }^{6}$

All automated activities must be scheduled and uplinked in accordance with satellite and ground constraints while respecting the overall available onboard resources. One of the tasks of the LMS is to provide sufficient onboard autonomy such that in case of a ground anomaly, the EDRS payload is still given an autonomy period of at least 8 hours. Flight procedures are therefore typically commanded time-tagged and eight hours in advance of their execution.

Onboard resources like master timeline slots are sparse, especially for EDRS-A with its E3000 satellite platform. To provide maximum autonomy and react timely to problems onboard, these resources need to be utilized to full capacity. The LMS is therefore dependent on timely feedback from telemetry and does not rely solely on on-ground modeling.

The timeline and high-level requests received from MOC are usually not static. Re-scheduling of links on MOC side entails a sequence of automated activities on DPCC side. This includes automated switching of payload configuration, deletion and re-commanding of parts of the uplinked mission time-line.

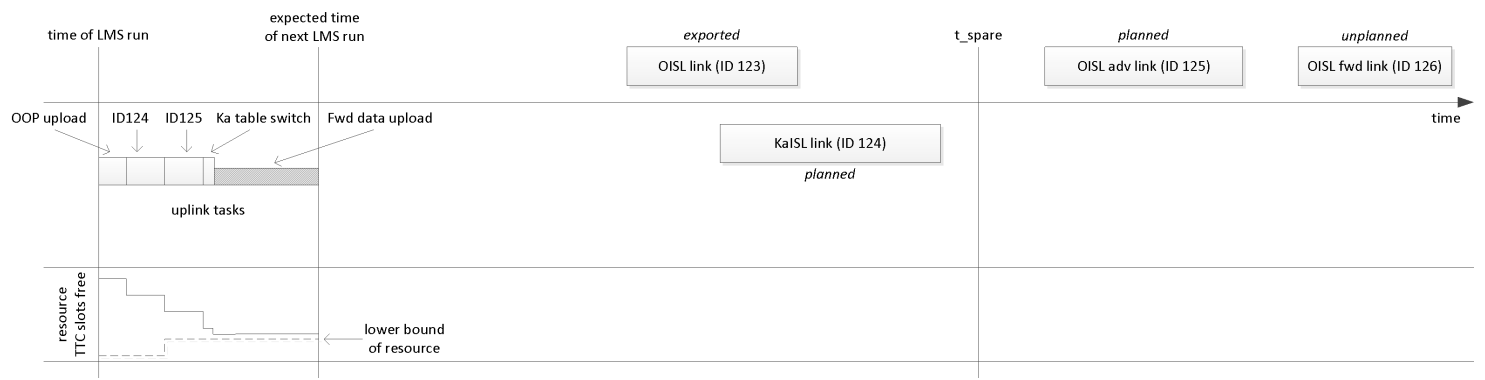

Figure 3: Schematic timeline after an LMS scheduling run: in this fictitious example, two additional link sessions are going to be uploaded (ID 124, 125) plus some amount of forward data. The procedure for link session 126 could not yet be uplinked due to the number of available time-tag slots

\section{Payload Related Maintenance Tasks}

In addition to the request based scheduling of payload activities, the DPCC is responsible for the execution of automated payload maintenance tasks. There are four main types of payload related tasks:

LCT time synchronization: for EDRS-A, the LCT hosts its own onboard clock which is not automatically synchronized with and has to maintain a better precision than the onboard clock of the satellite platform. To ensure successful link execution, the LCT clock must be correctly set within a maximum tolerance of $\pm 500 \mathrm{~ms}$ while the clock itself experiences a typical jitter of $100 \mathrm{~ms}$. Additional operational constraints need to be respected: a synchronization must not be performed during link execution, for obvious reasons. To account for possible commanding delays, the limit defined in the operational concept before an automated clock synchronization is triggered amounts to $250 \mathrm{~ms}$. Any systematic clock drift measured in telemetry is analyzed autonomously and accounted for in the synchronization process.

OOP upload: the LCT also maintains its own onboard orbit propagator (OOP) which needs to be updated in regular intervals. The OOP products are required during the initial pointing phase of an intersatellite link, before the acquisition of the counter terminal. The validity of each individual OOP is 4 hours and since the execution of an OOP update must not occur during link execution, special considerations need to be taken into account in case a link duration exceeds 4 hours, which in certain use-cases is possible. 
Alignment matrix calibration: the optical payload is submitted to harsh environmental influences and compensates for that by use of an onboard LCT alignment matrix. This alignment matrix needs to be re-calibrated in regular intervals, latest every 14 days. For the calibration to be successful, it is necessary to collect attitude related telemetry during every link session. This telemetry is automatically compiled by the automation engine and provided to flight dynamics, taking care of the necessary evaluations and sending the data back to the LMS for scheduling a calibration of the alignment matrix. Additional constraints need to be respected for this activity, i. e. the LCT needs to be in parked position. The calibration therefore is typically performed during station keeping maneuvers.

Ka-antenna pointing table management: in order to track the LEO target during an RF intersatellite links, Ka-antenna steering is managed using two onboard pointing tables, each table holding up to 1440 pointing records. Of these two tables, one can be set active and is henceforth read-only while the other remains inactive and editable. The tables are populated by the LMS, taking into account any constraints on pointing and steering velocity.

To allow for dynamic scheduling, three basic Ka-table operations need to be implemented in the Link Management System, as depicted in Figure 4: for additional link requests, new records must be added to the inactive table. In case links are removed from the timeline, the inactive table has to be populated with all subsequent pointing records and an updated rallying record to connect the adjacent links. Link additions are treated similar, the rallying of the adjacent link needs to be adapted as well. After any of these elementary operations, a time-tagged table switch is to be scheduled to ensure continuous steering of the Ka-antenna.

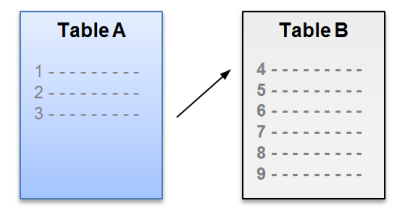

(a) Link addition

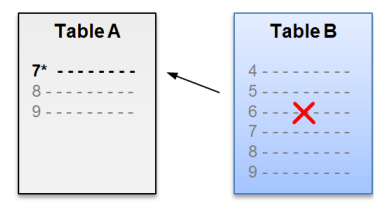

(b) Link deletion

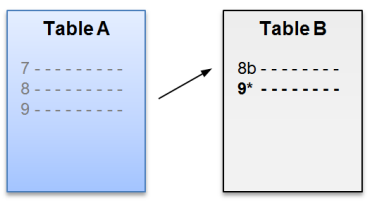

(c) Link insertion

Figure 4: Ka-table management: for each operation, the currently active table (in blue) must be switched.

All these activities are executed fully autonomously by the Link Management System in connection with the automation engine.

\section{Automated Request Execution}

The automation engine has direct access to the Flight Operations Procedure (FOP) repository and interfaces with the core Monitoring and Control system. The MCS interface, which is realized via network socket calls, allows access to the telemetry processor, the telecommand releaser and other systems used for reaction monitoring, like the TC History.

Once an external or internal request has been fully processed and scheduled by the Link Management System, an automation request is sent to the automation engine. The latter automatically calls a predefined Flight Operations Procedure (FOP) and the corresponding telecommands (TCs) are instantiated with parameter values taken from the automation request.

For EDRS-A, telecommands are sent both to the MCS and to a protocol converter, generating TC Set files (in JSON format) which are sent via file interface to the Eutelsat SCC to be uplinked to the spacecraft. It should be mentioned that for EDRS-A, the dispatch of telecommands to the GSOC MCS only serves the purpose of shadow commanding, required for processing command acknowledgments from telemetry and assigning them to the respective telecommands. The actual commanding of the payload is performed via the Eutelsat SCC on basis of the generated TC Sets. For EDRS-C, the TC Set generating module will be deactivated, so the same implementation can be used for both missions.

Every Flight Operations Procedure contains not only telecommands but a set of telemetry statements 
which are scheduled such that the payload state is verified against a set of pre- and post-conditions. The automation engine processes the combination of SCC $\log$ files, command execution verification and telemetry checks to correctly evaluate the execution state of a given activity and its associated request. The result of this evaluation is automatically logged and reported back to the Mission Operations Center.

In summary, the automation engine takes care of the following tasks:

- Instantiation of Flight Operations Procedures

- Conversion of ground time to onboard time using the time correlation provided by the MCS

- Generation of TC Sets, dispatch to the Eutelsat SCC and analysis of the returned TC Set logs

- Scheduling and execution of flight procedure defined telemetry checks

- Monitoring of onboard events related to telecommand execution

- Overall monitoring of the execution state of flight procedures

- Monitoring of the onboard queue and correlation with the on-ground model

- Real-time reporting of the onboard queue back to the Link Management System

- Monitoring of the LCT's GPS clock and triggering of synchronization requests

- Reporting of the general LCT and Ka-payload's health status

- Generation of link and LCT alignment related telemetry reports

- User interface for automation control, request monitoring and simple anomaly handling

One of the more intricate tasks in the implementation was the derivation of request states from the states of their associated sub-tasks. For the automation engine, each request can consist of several procedures which themselves consist of a sequence of telecommands and telemetry checks. However some procedures, like Ka-table record uploads, cannot be associated with a single request but have impact on several requests. On the other hand, the interface to the Mission Operations Center allows only two possible outcomes for any given link request, it is either successful or failed. A careful analysis was therefore necessary on how Flight Operations Procedures need to be designed in order to allow autonomous execution and state verification. Since automated systems may at times jump to hasty conclusions, a balance had to be found between timely reporting to the MOC and allowing the system to react on updated information or an operator to intervene in case of anomalies, while still maintaining the correct result in the reporting to the MOC. ${ }^{7}$

\section{E. Reporting}

Due to the high degree of capacity utilization and the number of involved parties, special attention was given to the design of automated reporting capabilities. A large number of external interfaces had to be implemented in order to meet all requirements, see also Figure 2. Telemetry analysis and key performance indicators are provided in quasi real-time to the Mission Operations Center via web- and file interface. This includes confirmations of reception, scheduling, uplink and execution of payload configuration requests as well as link session analysis and continuous reporting of the payload's main status. Possible issues during the execution of payload requests had to be taken into consideration beforehand, such that the automated reporting provides comprehensible information, not only to external parties but also for internal analysis.

The real-time reporting is complemented by long term telemetry analysis which can be either provided automatically or on-request. For this purpose, GSOC uses an in-house adaptation of the ROOT framework ${ }^{4}$ named RootVis which allows processing of very large datasets and the detection of outliers and anomalies in those datasets. 


\section{Situational Awareness and Anomaly Handling}

Each software component taking part in the DPCC automation chain interfaces with a central network and process monitor, a GSOC multi-mission software component called Nemo. Each application reports its health status and any information that is of potential interest to the operator in order to provide a central man-machine interface for system monitoring. During the EDRS routine phase, operators are notified only in case of an anomaly. Apart from that the system is fully autonomous. Therefore it is of the utmost importance that in case of an anomaly, the operator can gain a full overview of request, payload, and ground-segment status, as fast as possible.

Nemo therefore offers a graphical user interface, very much like other commercial monitoring solutions, but with a set of proprietary interfaces allowing the system to receive comprehensive status updates from the DPCC software components. The monitoring tool distinguishes between routine logging, the occurence of warnings and errors and the generation of alarms, which can be audible, visual or via email notification. As soon as an operator is notified and is aware of the situation, he can start recovery actions for the affected components. The operational concept foresees the operator to solve simple problems on his own, following strictly defined actions specified in ground operations procedures (GOPs). In case the problem cannot be solved in this way, the operator notifies the on-call satellite support or system engineer who can arrive on-site in less than an hour.

\section{A. Anomaly Handling}

In case of an anomaly, it is important for the operator to pause the automation engine either controlled or abruptly, depending on the nature of the problem. For problems of high severity, automated commanding can be brought to an immediate halt, thereby giving the operator time to further analyze the situation and start a manual recovery.

It should be noted that manual operations still uses part of the automation chain in order to benefit from the enhanced monitoring, commanding and reporting capability the system offers. Flight procedures and recommendations can be manually instantiated and executed via a GUI component in the generic procedure generator (GPG), even while the processing of automated requests is stopped. The GPG routes its output via the Automator to Eutelsat SCC and to SCOS. Telemetry checks are performed autonomously, as they would be for automated requests. This concept of manual operations is used extensively during early operations, IOT and commissioning. ${ }^{7}$

\section{B. Redundancy Concept}

The DPCC redundancy concept is based on a three processing chain setup. All three chains are set up identically and only one branch is needed to fulfill the functional requirements. In order to provide maximum redundancy and seamless operations, one chain typically is dedicated to be in hot-stand-by, ready to take over in case of hard- or software failure on the active chain. The third chain is used in the frame of simulations, tests and software upgrades.

The chains are composed of stand-alone software components tied together by an automated file distribution system (AFD) with minimum set of cross-connections between chains. Any implemented software per se has no information about the chain it is allocated to or the role of that chain.

Since the communication between DPCC components is file based for the most part, redundancy switching is realized via the use of symbolic links in the file system. These links can be switched between actively populated directories and a dead directories. A central software component allows the operator to view and change the switch settings of all chains in a single step. 


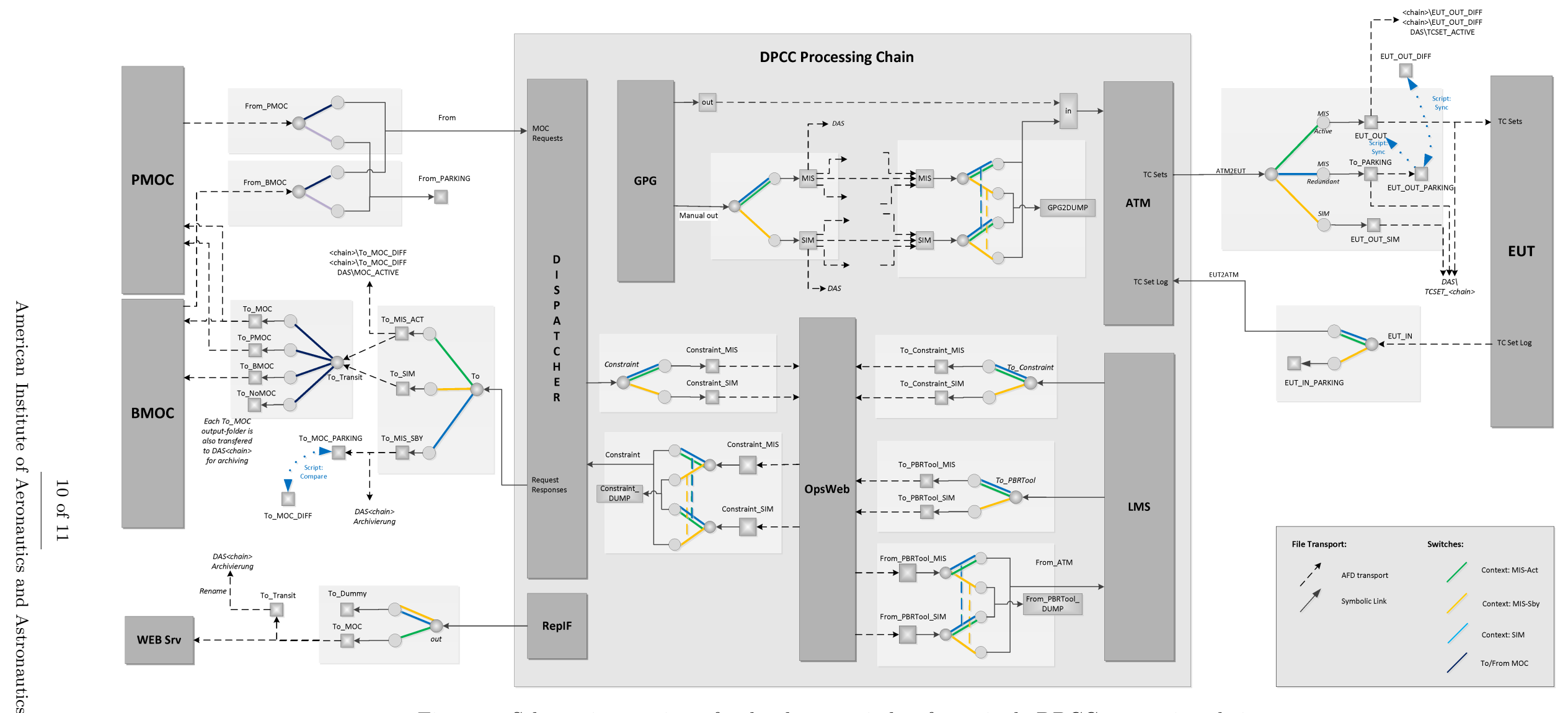

Figure 5: Schematic overview of redundancy switches for a single DPCC processing chain 


\section{Conclusion}

The payload and satellite operations concept of the EDRS constellation poses both challenges and opportunities to the ground segment design. DLR GSOC has leveraged the design and implementation of the DPCC ground segment and early operations of the EDRS-A payload. DLR is now in a position to reuse and extend the largest part of the system design for the EDRS-C ground segment implementation, which is currently ongoing. The mission integrates well with the GSOC multi-mission approach and a large part of operational concepts could be streamlined by the deployment of automation, resulting in a reduction of project costs and operational overhead.

\section{References}

\footnotetext{
${ }^{1}$ Wallrapp, F., Ballweg R. and Gataullin, Y, The European Data Relay System (EDRS) Operational Challenges, IAC11.B6.2.4, 2011

${ }^{2}$ ECSS Secretariat, Ground systems and operations - Telemetry and telecommand packet utilization, Space Engineering, No. ECSS-E-70-41A, ESA-ESTEC, 2003

${ }^{3}$ Stangl, C., Braun, A., Geyer M.P., GECCOS - the new Monitoring and Control System at DLR-GSOC for Space Operations, based on SCOS-2000, SpaceOps 2014 Conference, SpaceOps Conferences, (AIAA 2014-1602)

${ }^{4}$ Brun, R., Rademakers, F., ROOT - An Object Oriented Data Analysis Framework, Proceedings AIHENP'96 Workshop, Lausanne, Sep. 1996, Nucl. Inst. \& Meth. in Phys. Res. A 389 (1997) 81-86. See also http://root.cern.ch/

${ }^{5}$ Perera, N., Automatic Config Management - Autodiscovery of Configuration Items and Automatic Configuration Verification, SpaceOps 2016 Conference, Daejeon, Korea (submitted for publication)

${ }^{6}$ Göttfert, T., Grishechkin, B., Wörle, M.T., Lenzen, C., The Link Management System for the European Data Relay System, SpaceOps 2016 Conference, Daejeon, Korea (submitted for publication)

${ }^{7}$ Scharringhausen, J.C., Kolbeck, A., Beck, T., A Robot on the Operator's Chair - The Fine Line Between Automated Routine Operations and Situational Awareness, SpaceOps 2016 Conference, Daejeon, Korea (submitted for publication)
} 